\title{
Paraplegia by Acute Cervical Disc Herniation after Shoulder Arthroscopic Surgery in Beach-Chair Position
}

\author{
Hye Young Kim, Gang Han Jung \\ Anesthesiology and Pain Medicine, College of Medicine, Chungju Hospital, Konkuk University, Seoul, South Korea \\ Email: hae1127@kku.ac.kr
}

How to cite this paper: Kim, H.Y. and Jung, G.H. (2018) Paraplegia by Acute Cervical Disc Herniation after Shoulder Arthroscopic Surgery in Beach-Chair Position. Open Journal of Anesthesiology, 8, 280-283.

https://doi.org/10.4236/ojanes.2018.811028

Received: September 14, 2018

Accepted: November 12, 2018

Published: November 15, 2018

Copyright $\odot 2018$ by authors and Scientific Research Publishing Inc. This work is licensed under the Creative Commons Attribution International License (CC BY 4.0). http://creativecommons.org/licenses/by/4.0/

\begin{abstract}
Paraplegia after non-cervical spine surgery under general anesthesia is a rare devastating postoperative complication [1]. A patient with shoulder pain was scheduled for shoulder arthroscopy due to rotator cuff repair. Arthroscopic shoulder surgery was performed with the patient in the beach-chair. Postoperatively, patients complained the weakness of both lower leg and marked numbness and tingling in the both arms. MRI showed a herniated intervertebral disc between C6 and C7 with spinal cord compression. Despite urgent discectomy, the patient remained dysesthesia of both upper extremities for 6 months. The purpose of this report is to introduce our case with a paraplegia observed after arthroscopic shoulder surgery in beach-chair position because of acute cervical herniation.
\end{abstract}

\section{Keywords}

Beach Chair Position, Paraplegia, Shoulder Arthroscopy

\section{Introduction}

Paraplegia after general anesthesia is a rare postoperative complication. Mechanical or ischemic injury is ascribed to be the most common etiology [2]. We present a case of acute paraplegia after arthroscopic shoulder surgery in beach-chair position.

\section{Case Report}

A 53-year-old man with a 3-month history of increasing right shoulder pain was scheduled for right shoulder arthroscopy. His medication included amlodipine, atenolol, atorvastatin and metformin with history of controlled hypertension 
and diabetes mellitus.

He had no symptoms in the neck or opposite arm. Pre-operative laboratory findings were normal. On the airway examination, he showed no limitation in his neck movement. The patient's blood pressure was 130/80 $\mathrm{mmHg}$ before induction of anesthesia. Anesthesia was induced propofol $120 \mathrm{mg}$ and rocuronium $50 \mathrm{mg}$ and maintained with desflurane $4.0-6.0$ vol\% in $\mathrm{O}_{2} / \mathrm{N}_{2} \mathrm{O}$. The trachea was easily intubated without overextension of the neck. The patient was placed in the beach-chair position; his head was stablilized and the upper limb immobilized with the traction. The systolic blood pressure was maintained $90 \mathrm{mmHg}$ and $140 \mathrm{mmHg}$ during anesthesia. When the systolic blood pressure was 90 $\mathrm{mmHg}$, phenylephrine $50 \mathrm{ug}$ was given intravenously. Intravenous fentanyl 50 ug was administered $30 \mathrm{~min}$ before the end of surgery.

Arthroscopic subacromial decompression and rotator cuff repair was performed. The operation lasted 3 hours 30 minutes, and no complications occurred intraoperatively. The recovery process was smooth and the patient was transferred to ward.

About 2 hrs after anesthesia, the patient complained the weakness of both lower leg and marked numbness and tingling in the both arms. An emergency MRI showed aintervertebral disc herniation between C6 and C7 with spinal cord compression. No epidural hematoma or other abnormal were observed (Figure 1). Judging from the clinical symptoms and MRI finding, the patient was diagnosed with paraplegia caused by cervical disc herniation. Several hours after the acute onset of paraplegia, the patient underwent urgent anterior approach with discectomy and anterior internal fixation. Muscle contraction of lower limbs was not observed immediately postoperatively. On postoperative day 1 , muscle testing for the right leg was normal and left leg was fair. Muscle testing was normal for both legs on postoperative day 2. However, numbness and tingling in the both arms was sustained. Patient discharged at 40 days after shoulder arthroscopy. After 6 months, the patient remained dysesthesia of both upper extremities.

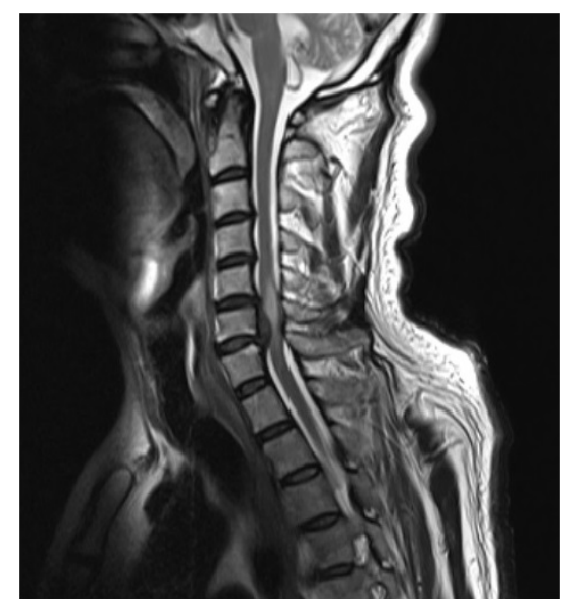

Figure 1. MRI demonstrates disc herniation at C6-C7 with spinal cord compression. 


\section{Discussion}

Complications related to patient positioning and anesthesia for shoulder procedures have received recent attention. Athroscopy of the shoulder was first described by use of the lateral decubitus position to allow for traction at the glenohumeral articulation. The beach chair position (BCP) was developed to improve access for shoulder arthroscopy procedures. The position presents the anatomy in a beach-chair position, decreases distortion of the intra-articular anatomy, minimizes spatial disorientation, and offers easy conversion to an open procedure when necessary [3] [4].

Maintaining a safe position for the head during shoulder surgery can be challenging, particularly in the beach-chair position when universal positioners are used to place the arm into traction. In beach-chair position, hyperextension and rotation or tilt of the head can decrease vertebral artery blood flow causing infarcts of the posterior vertebral artery circulation. In addition, an ischemic event caused by air embolism is of greater theoretical risk to patients in beach-chair position [5]. However, there is no report that acute disc herniation developed after shoulder arthroscopy in beach-chair position. Excessive neck extension and rotation during endotracheal intubation and positioning could trigger the bulging disc in the preexisting undiagnosed cervical lesion because of the loss of muscle support by muscle relaxant given during general anesthesia [1]. Coexisting cervical spine disorders such as spondylosis, herniated disc, and spinal canal stenosis are not uncommon in the elderly patients. Previous reviewers have demonstrated a high incidence of concomitant cervical and lumbar spine diseases in patients without trauma history [6] [7].

Our patient had a back pain for several years and degenerative spinal stenosis at L1/2. Patients presenting with lumbar spine problems should be evaluate thoroughly on cervical spine before surgery. Positive findings in detailed physical examination of neck require additional neurological or radiological examinations. Pain and paresthesia can be sought during the movement. Because instability of cervical spine is usually asymptomatic, care must be taken in order to maintain the neck in the neutral position during airway maneuvering and positioning.

Determining diagnosis on acute spinal cord compression during general anesthesia appears to be difficult because patients who are still under sedation are unable to complain any discomfort on the head and neck regions. In addition, skillful intubation and careful positioning of head and neck are mandatory for patients in the beach-chair position.

\section{Conclusion}

In conclusion, paraplegia after non-cervical spine surgery under general anesthesia is a devastating complication, which often results in permanent disability or neurological deficit. We should pay attention to this complication for shoulder arthroscopic surgery. Excessive neck movement is believed to be triggering 
factor and skillful intubation and neck supporting are recommended to reduce cervical cord injury. And anesthesiologists and surgeons should be aware of this complication and diagnosis should be promptly made for early interventions.

\section{Informed Consent}

Informed consent for publication was obtained from patient.

\section{Conflicts of Interest}

The authors declare no conflicts of interest regarding the publication of this paper.

\section{References}

[1] Wakim, E. and Beaufils, P. (1991) Arthroscopy of the Shoulder with the Patient in Beach-Chair Position. Revue de Chirurgie Orthopédique et Réparatrice de 1 Appareil Moteur, 77, 577-580.

[2] Johann, J.L., Martin, O., Alois, O. and Stephan, F. (1996) Quadriplegia after Lumbar Disc Surgery: A Case Report. Spine, 21, 1932-1935. https://doi.org/10.1097/00007632-199608150-00021

[3] Afshinmajd, S., Khalaj, A., Roohani, Y., Yarmohammadi, M., Salehpour, S., Saeedi, A., Ghaedi, G.H. and Seradj, M.H. (2011) Acute Paraplegia after General Anesthesia. Acta Medica Iranica, 49, 560-564.

[4] Peruto, C.M., Ciccotti, M.G. and Cohen, S.B. (2009) Shoulder Arthroscopy Positioning: Lateral Decubitus versus Beach Chair. Arthroscopy, 25, 891-896. https://doi.org/10.1016/j.arthro.2008.10.003

[5] Pohl, A. and Cullen, D.J. (2005) Cerebral Ischemia during Shoulder Surgery in the Upright Position: A Case Series. Journal of Clinical Anesthesia, 17, 463-469. https://doi.org/10.1016/j.jclinane.2004.09.012

[6] Jacobs, B., Ghelman, B. and Marchisello, P. (1990) Coexistence of Cervical and Lumbar Disc Disease. Spine, 15, 1261-1264. https://doi.org/10.1097/00007632-199012000-00006

[7] Teng, P. and Paoatheodorou, G. (1964) Combined Cervical and Lumbarpondylosis. Archives of Neurology, 10, 298-307.

https://doi.org/10.1001/archneur.1964.00460150068007 